\title{
La movilidad sostenible como estrategia para el fortalecimiento de la educación ambiental
}

\section{Sustainable mobility as a strategy for strengthening environmental education}

\author{
Ángel Darío Díaz-Cordero \\ diazcordero.28@gmail.com \\ Universidad Popular del Cesar, Valledupar \\ Colombia \\ https://orcid.org/0000-0002-7655-9674 \\ José Francisco Marimón-Lozano \\ gordomarimon@hotmail.com \\ Universidad Popular del Cesar, Valledupar \\ Colombia \\ https://orcid.org/0000-0002-9278-9189 \\ María-del-Carmen Jiménez-Barriosnuevo \\ mariacjimenez@unicesar.edu.co \\ Universidad Popular del Cesar, Valledupar \\ Colombia \\ https://orcid.org/0000-0002-2491-3731
}

Recibido: 15 de abril de 2021 Aprobado: 05 de julio de 2021 


\title{
RESUMEN
}

Es evidente la necesidad de fortalecer la formación de una Cultura Ambiental con nuevos enfoques y estrategias más holísticas, donde la Educación Ambiental se constituya en eje dinamizador del proceso de aprendizaje. Considerando esto, la investigación se enmarcó en describir cómo perciben los estudiantes de la Institución Educativa "EI Carmen" de Colombia, la movilidad sostenible como principio pedagógico transversal desde la Educación Física. Metodológicamente la investigación se cimiento desde un enfoque cuantitativo, en correspondencia con un diseño no experimental, de campo, a nivel descriptivo y de corte transversal. La muestra estuvo conformada por 16 estudiantes. Finalmente, la movilidad sostenible se constituye en un enfoque pedagógico innovador que puede ser implementado en el marco el Proyecto Ambiental Escolar, mediante el desarrollo de ciclopaseos y el uso de la bicicleta, para la formación de ciudadanos con conciencia ambiental críticos y exigentes con la protección y el desarrollo sostenible del entorno.

Descriptores: Educación ambiental; educación física; educación para el desarrollo sostenible. (Palabras tomadas del Tesauro UNESCO).

\begin{abstract}
The need to strengthen the formation of an Environmental Culture with new approaches and more holistic strategies is evident, where Environmental Education becomes the driving force behind the learning process. Considering this, the research was framed in describing how the students of the Educational Institution "El Carmen" in Colombia perceive sustainable mobility as a transversal pedagogical principle from Physical Education. Methodologically, the research was founded from a quantitative approach, in correspondence with a non-experimental, field design, at a descriptive and cross-sectional level. The sample consisted of 16 students. Finally, sustainable mobility constitutes an innovative pedagogical approach that can be implemented within the framework of the School Environmental Project, through the development of cycle paths and the use of bicycles, for the training of environmentally conscious citizens who are critical and demanding of protection. and the sustainable development of the environment.
\end{abstract}

Descriptors: Environmental education; physical education; education for sustainable development. (Words taken from the UNESCO Thesaurus). 


\section{INTRODUCCIÓN}

Las interacciones habitantes de ciudad/entornos naturales, generalmente ocasionan impactos ambientales negativos desde el momento en que se rompe la comunicación entre las actividades diarias realizadas por las personas con las dinámicas de los ecosistemas; así, su estado se deteriora al incrementarse la presencia de la comunidad en estos espacios.

En este contexto, uno de los desafíos más significativos relacionado con la contaminación del medio ambiente reside en la movilidad, pues, en las ciudades existe un número indefinido de vehículos y sistemas de transporte para el normal desplazamiento de la población. Es por ello, que fácilmente un tercio de las emisiones mundiales de $\mathrm{CO}_{2}$ provienen de los medios de transporte de personas, razón por la cual, la búsqueda de posibles soluciones de transporte con bajas emisiones de carbono representa uno de los retos más relevantes en favor del medio ambiente, los recursos naturales y su desarrollo sostenible (Arellana, Puche \& García, 2019).

De allí que es comprensible el hecho de que conservar y preservar el medio ambiente no solamente es responsabilidad de los especialistas en Ciencias Naturales, por lo contrario, requiere de la concurrencia de todos los habitantes del planeta (Nyambe, 2012). Por consiguiente, el ser humano viene asumiendo una mayor conciencia y actitud positiva hacia la defensa y conservación del medio ambiente en general y de su entorno próximo en particular, tratando de cooperar en la resolución de los principales problemas ambientales generados por la sociedad moderna (Sáez, 2012).

De los anteriores planteamientos se deduce que, la movilidad sustentable debe ser una política prioritaria en los gobiernos y una responsabilidad estricta de los ciudadanos, sería la manera más adecuada de colaborar en beneficio del medio ambiente. Representa igualmente un modelo de movilidad que no da origen a impactos negativos sobre las condiciones del medio ambiente, y, por el contrario, se identifica plenamente con el bienestar y calidad de vida de las personas. De ahí la importancia de enfatizar sobre el 
hecho de que la movilidad sostenible es una postura que permite aprovechar las posibilidades de transportarse sin necesidad de sacrificar el entorno y los recursos, que representan un capital inigualable para las próximas generaciones (Sanz, 2016).

En este sentido, una alternativa viable es la inversión en Educación Ambiental (EA), que proporcionaría a los ciudadanos de todos los grupos etarios, acceso a información que los posibilita a entender la limitación de los recursos naturales en el planeta. Más aún, si se asume desde una cosmovisión que promueva, desde las actividades físico-deportivas, una mejor simbiosis individuo/entorno en todos los ambientes naturales (De Maio, Linório \& Tûrk, 2018).

Históricamente se sabe que la Organización de las Naciones Unidas para la Educación la Ciencia y la Cultura (UNESCO), ha realizado y sigue realizando esfuerzos por estudiar y promover las formas de incluir el tema ambiental como recurso formativo, considerando que la EA debe ser diseñada y ejecutada desde las instituciones educativas (Martínez, 2017).

En este tenor, resulta oportuno destacar que, considerando la Ley 99 de 1993 y la Ley 115 en conjunto con el Decreto 1743, ambos de 1994, en las instituciones educativas de la República de Colombia, la EA juega un papel preponderante en los procesos formativos y ambientales; pues, con ella se promueve la comprensión de las relaciones de interdependencia y los modos de actuar de los sujetos sobre el entorno biofísico y socio-cultural. Entendido esto, como forma de conocimiento para la transformación de la realidad y el desarrollo de actitudes de valoración y respeto por el ambiente, lo que beneficia la pertinencia e implementación de los Proyectos Educativos Institucionales (PEI) y, por ende, la calidad de la educación (MEN \& MADS, 2002).

La EA es una estrategia flexible, que tiene un tejido de propósitos y procesos en permanente construcción (Pulido \& Olivera, 2018). Pues, religar al ser humano con los ecosistemas que hacen parte del territorio habitado de manera favorable, requiere del desarrollo de sus capacidades para percibir los mensajes que cotidianamente le 


\section{CIENCIAMATRIA}

Revista Interdisciplinaria de Humanidades, Educación, Ciencia y Tecnología

Año VII. Vol. VII. N¹. Edición Especial. 2021

Hecho el depósito de ley: pp201602FA4721

ISSN-L: 2542-3029; ISSN: 2610-802X

Universidad Nacional Experimental Francisco de Miranda (UNEFM). Santa Ana de Coro. Venezuela

Ángel Darío Díaz-Cordero; José Francisco Marimón-Lozano; María del Carmen Jiménez-Barriosnuevo

proporciona la naturaleza y convertir esta experiencia dinámica en procesos de formación, donde se problematice la realidad y se, identificar comportamientos a transformar (Wilches, 2013).

Al respecto, Torres \& Velandia (2013), consideran que la EA convierte la vida misma en maestra, permitiendo al individuo comprender las relaciones de interdependencia con su entorno a partir de procesos de reflexión-acción permanentes en su cotidianidad, con el fin de construir propuestas de apropiación territorial que le permitan establecer relaciones con la comunidad y la naturaleza

Es por ello que, en las últimas décadas, múltiples experiencias de EA han tratado de contribuir a la solución de problemas ambientales. En tal sentido, han buscado fomentar transformaciones culturales en las comunidades locales, tomando en cuenta las particularidades propias del contexto y bajo una directriz clara en cada uno de los programas, En efecto, implica mejorar la relación entre los seres humanos y la naturaleza, a partir de transformar comportamientos que degradan los entornos naturales (Páramo, 2009).

Es de saber que en las instituciones educativas la EA, debe estar regida por los principios de: interculturalidad; formación en valores; regionalización; interdisciplinariedad; participación y formación para la democracia, la gestión y la resolución de problemas. Además, debe estar presente en todos los componentes del currículo, mediante prácticas transversales, entendidas estas como la integración de las diversas disciplinas en torno de un propósito común. Pues, la transversalidad en la Educación propicia la gestión del conocimiento desde una perspectiva más integradora y desde ambientes de aprendizaje más dinámicos (González, 2014).

Considerando la proposición de la movilidad sostenible desde este contexto de la transversalidad educativa, cabe considerar a la Educación Física (EF) como ámbito para ello. De hecho, la EF abrazada a la movilidad sostenible ubica al estudiante en distintos lugares en el espacio con un objetivo humano, social y académico definido, donde la 


\section{CIENCIAMATRIA}

Revista Interdisciplinaria de Humanidades, Educación, Ciencia y Tecnología

Año VII. Vol. VII. N¹. Edición Especial. 2021

Hecho el depósito de ley: pp201602FA4721

ISSN-L: 2542-3029; ISSN: 2610-802X

Universidad Nacional Experimental Francisco de Miranda (UNEFM). Santa Ana de Coro. Venezuela

Ángel Darío Díaz-Cordero; José Francisco Marimón-Lozano; María del Carmen Jiménez-Barriosnuevo

disposición, motivación y compromiso de este, lo hacen más propenso y sensible al conocimiento, que cuando se encuentra en el ambiente cotidiano de aprendizaje afianzado en el salón de clases.

La EF abarca muchos aspectos, pero, podría decirse que cuando se habla de esta rama de la Educación, se hace referencia a la disciplina que se relaciona con el uso total de todo el cuerpo y que pedagógicamente permite la formación integral de los individuos. En el marco de este planteamiento, cabe resaltar que la actividad físico-deportiva representa una práctica cotidiana que se viene ejerciendo cada vez más en sus diversas modalidades. Este incremento es ocasionado por diversas situaciones relacionadas con las dinámicas sociales como la necesidad de mejorar el aspecto físico, los estilos de vida saludables y hasta la moda. No obstante, esto, necesariamente implica el uso, por parte de deportistas y/o aficionados, de áreas naturales para llevar a cabo las actividades, lo que lleva implícito la necesidad y responsabilidad de conservar, proteger y preservar el ambiente (Legido-Díez, Ruiz, Brito \& Navarro, 2008).

En atención a lo expresado, es pertinente considerar a Luque, Baena \& Granero (2011), cuando sugiere que toda actividad que se realiza en el ambiente, genera diversos impactos y la práctica de actividades físico-deportivas en medios naturales no se escapa de esta realidad. Si bien es cierto que estas prácticas físico-deportivas en medios naturales, coadyuvan a la promoción de espacios naturales a nivel local, del turismo deportivo, convirtiéndose en vehículos de desarrollo social. No es menos cierto que, también, pueden ser generadoras de daños ecológicos que pueden afectar, en mayor o menor grado, los ecosistemas, dada la acumulando de residuos sólidos, productora de contaminación.

Entonces, utilizar cada vez más los espacios naturales para convertirlos en zonas de recreación y para la práctica de actividades físico-deportivas, significa que la naturaleza está perdiendo la propiedad de ser virgen, abriéndole las puertas a generaciones de 


\section{CIENCIAMATRIA}

Revista Interdisciplinaria de Humanidades, Educación, Ciencia y Tecnología

Año VII. Vol. VII. N¹. Edición Especial. 2021

Hecho el depósito de ley: pp201602FA4721

ISSN-L: 2542-3029; ISSN: 2610-802X

Universidad Nacional Experimental Francisco de Miranda (UNEFM). Santa Ana de Coro. Venezuela

Ángel Darío Díaz-Cordero; José Francisco Marimón-Lozano; María del Carmen Jiménez-Barriosnuevo

personas que probablemente aún no están educadas para valorar los recursos naturales y los ecosistemas (Minambiente, 2002). De allí la importancia

de tener claridad sobre el hecho de que las actividades en las que se interactúa con la naturaleza deben estar planteadas dentro de un marco de respeto y protección del medio ambiente, razón por la cual, se debe contar con el conocimiento y las herramientas que permitan una correcta toma de decisiones a la hora de implementar cualquier estrategia en favor del medio ambiente (Baena \& Granero, 2008).

Así lo ratifica Lagardera (2009), cuando declara que teniendo en cuenta los efectos en el paisaje natural que pueden ocasionar tanto las actividades físicas y recreativas, como la infraestructura y equipamientos públicos ubicados para mejorar el desarrollo de la actividad deportiva, es imperativo crear conciencia sobre la importancia de velar por el cuidado y la preservación del medio ambiente.

Considerando lo dicho, se asume a la escuela como entidad para la creación de una cultura medioambiental en virtud del cumplimiento de su función educadora para generar cambios y transformaciones que requiere la sociedad. Sin embargo, la realidad actual dista mucho de esto, aunque se le atribuya a la Educación la función mediadora entre las prácticas humanas y el uso consciente del entorno, como ya se enuncio (Flórez, 2012). Muestra de ello, en Colombia, pese a los esfuerzos realizados por el MEN y el Ministerio de Ambiente y Desarrollo Sostenible, se evidencian dificultades en la aplicación de la política de EA. En tal sentido, desde lo inscrito por Flórez (2012), resalta que no existe una conceptualización clara con respecto al ambiente y la EA, aunado a la desarticulación que se da entre el PEI y el Proyecto Ambiental Escolar (PRAE) y que se refleja en la estructura curricular, pues no se acoge la Educación Ambiental como eje transdisciplinar y de discusión permanente en los procesos formativos.

Asimismo, en el municipio de Cotorra, Departamento de Córdoba y específicamente en los alrededores de la Institución Educativa El Carmen, las políticas ambientales establecidas por los entes gubernamentales y municipales para la protección y 


\section{CIENCIAMATRIA}

Revista Interdisciplinaria de Humanidades, Educación, Ciencia y Tecnología

Año VII. Vol. VII. N¹. Edición Especial. 2021

Hecho el depósito de ley: pp201602FA4721

ISSN-L: 2542-3029; ISSN: 2610-802X

Universidad Nacional Experimental Francisco de Miranda (UNEFM). Santa Ana de Coro. Venezuela

Ángel Darío Díaz-Cordero; José Francisco Marimón-Lozano; María del Carmen Jiménez-Barriosnuevo

preservación del medio ambiente son visiblemente deficiente. Pues, en observaciones realizadas en el mes de marzo de 2021 a razón de las actividades pedagógico-didácticas enmarcadas en el PRAE de dicha institución, se pudo percatar que algunos caminos y espacios naturales son convertidos en zonas para arrojar todo tipo de residuos sólidos, o igualmente áreas en las que se evidencia la tala indiscriminada de vegetación o quema de la misma, quedando al descubierto la falta de autoridad y de políticas serias por parte de estos entes que tienen la autonomía para sancionar a los posibles infractores.

Cabe agregar, que la realidad social de los moradores y de las zonas afectadas ameritan una posición más enérgica por parte del Estado hacia la comunidad y su entorno, por lo que se deben dar acciones de intervención ambiental más agresivas y contundentes, para evitar la contaminación y destrucción del medio ambiente.

De ahí, la importancia que tiene el hecho de implementar en los contextos educativos, nuevas estrategias pedagógicas desde la EF para fortalecer la EA, desde una visión más holística y transdisciplinar, tanto en estudiantes y docentes como en la comunidad educativa en general, a fin de que se conviertan en ciudadanos más críticos, exigentes, y veedores de acciones encaminadas a proteger el medio ambiente y velar por el desarrollo sostenible de su entorno y comunidad.

Promover el desarrollo de una Cultura Ambiental desde la EF transversalizada e integrada por los temas ambientales y en especial desde el marco de la movilidad sostenible es una vía prioritaria, considerando que este concepto emerge, y por tanto se ha de asumir, como alternativa para hacer frente a los grandes problemas medioambientales causados por el modelo de transporte urbano implantado desde mediados del siglo XX, la cual se caracteriza por el uso generalizado de automóviles de energía fósil altamente contaminante, que han deteriorado el medioambiente y, con ello, afectado tanto la calidad de vida de las personas, como el propio desarrollo socioeconómico de las ciudades (Herrera, 2018) 


\section{CIENCIAMATRIA}

Revista Interdisciplinaria de Humanidades, Educación, Ciencia y Tecnología

Año VII. Vol. VII. N¹. Edición Especial. 2021

Hecho el depósito de ley: pp201602FA4721

ISSN-L: 2542-3029; ISSN: 2610-802X

Universidad Nacional Experimental Francisco de Miranda (UNEFM). Santa Ana de Coro. Venezuela

Ángel Darío Díaz-Cordero; José Francisco Marimón-Lozano; María del Carmen Jiménez-Barriosnuevo

En todo este contexto de la movilidad sostenible, el uso educativo de la bicicleta se percibe como ámbito para el desarrollo de aprendizajes en torno al conocimiento del medio que rodea al ser humano y las problemáticas que este puede presentar. Al respecto, Luque (2016) \& Navarro (2014), platean que esta práctica posibilita la formación ciudadana para reconocer los entornos naturales y concuerdan con el hecho de que el uso de la bicicleta en el desarrollo de ciclo-paseos favorece diversas actitudes en el individuo como: el respeto de normas, cooperación, trabajo en equipo, creando igualmente hábitos saludables en relación con la salud física y mental a través del contacto directo con la naturaleza.

Lo anterior se cohesiona con lo planteado por Navarro (2014), quien considera que la percepción directa del espacio natural en la bicicleta potencia el desarrollo de valores para la convivencia entre las personas y los ecosistemas. De igual forma, permite analizar las trasformaciones del ambiente debido a diversas problemáticas que afectan el entorno natural como: la contaminación, la desforestación, explotación de recursos, ganadería extensiva, entre otros.

De todas maneras, lo realmente importante es que, desde la movilidad sostenible, los ciclo-paseos, además de ser una actividad físico-deportiva y recreacional que beneficia la parte física y mental del cuerpo, contribuye al desarrollo de una conciencia ambiental sustentada en valores éticos y responsabilidad con el medio. Siendo estas las razones más importantes por las cuales se está promoviendo de forma permanente el concepto movilidad sostenible en todos los países del mundo, incluido Colombia.

Aunque en la actualidad se pretende formar y crear conciencia en todos los seres humanos sobre su responsabilidad con el uso y mantenimiento de los espacios naturales, es posible la falta de EA en el contexto social colombiano. Por consiguiente, es pertinente utilizar todos los recursos didácticos y estrategias pedagógicas posibles con el propósito de apoyar y fortalecer un cambio comportamental de los estudiantes y de la comunidad 
en general para que valoren mucho más su interacción con la naturaleza, teniendo en cuenta la movilidad sostenible como una herramienta importante para lograrlo.

Finalmente, considerando que se busca desarrollar una investigación en el marco del PRAE de la Institución Educativa "El Carmen", en Cotorra, Departamento de Córdoba, de lo que representa la actividad físico-deportiva y recreativa, considerando que estas se pueden desplegar de manera transversal con la EA, desde la premisa de la movilidad sostenible mediante ciclo-paseos, se plantea la siguiente interrogante: ¿Cómo perciben la movilidad sostenible, los diferentes actores de dicha institución?

Entonces, con el propósito de responder este interrogante, se establece como objetivo de investigación, describir cómo perciben la movilidad sostenible, los estudiantes de la Institución Educativa "El Carmen" en Cotorra, Departamento de Córdoba, considerando que esta se puede asumir como principio pedagógico transversal para el fortalecimiento de la Cultura Ambiental desde la EF.

\section{METODOLOGÍA}

En este apartado se dilucida la metodología asumida para el desarrollo del proceso investigativo. No obstante, como ya se dijo, el estudio se desarrolla en el marco del PRAE de la Institución Educativa "El Carmen", como propuesta que busca que los estudiantes se motiven a asumir este principio y, por consiguiente, los ciclo-paseos no como una actividad meramente físico-deportivo y recreativa; sino, también, como espacio para aprender a reconocer y a actuar sobre las posibles circunstancias y riesgos que puedan afectar el medio ambiente.

En este contexto, y considerando lo planteado por Palella \& Martins (2015), la investigación se cimiento desde un enfoque cuantitativo y como estrategia para responder al problema de investigación se asumió un diseño no experimental, dado que no se manipuló deliberadamente ninguna variable Es de saber que la investigación, con este tipo de diseño, se caracterizó por ser de campo, a nivel descriptivo y de corte 
transversal, ya que, la recolección de los datos se hizo directamente de la realidad donde ocurren los hechos, en un momento único y con el propósito de describirlos e interpretarlos tal cual como ocurren.

Respecto a la muestra poblacional objeto de estudio, esta fue tomada de manera no probabilística e intencional, de acuerdo con los criterios definidos por los investigadores como: nivel y grado educativo, jornada matutina, grado de responsabilidad, aprobación de los padres, interés investigativo y disponibilidad para las actividades. En concreto, la muestra estuvo conformada por 16 estudiantes del octavo grado, de la jornada de la mañana.

Para hacer el trabajo de campo y recabar los datos de investigación se utilizó la técnica de la encuesta, mediante un cuestionario constituido por un conjunto de preguntas cerradas con múltiples opciones de respuesta. Asimismo, el procesamiento de los datos se hizo a través de la estadística descriptiva y se presentan mediante tablas y gráficos con sus respectivos análisis (Palella \& Martins, 2015). 


\section{RESULTADOS}

En este apartado, se presentan los resultados obtenidos con los cuestionarios aplicados a 16 estudiantes que hacen vida la Institución Educativa "El Carmen", con la intencion de describir perciben los ciclo-paseos, asumidos estos como herramienta pedagógica transversal de movilidad sostenible para el fortalecimiento de la cultura ambiental desde la EF. En tal sentido, se presenta los siguientes resultados:

Con respecto a cómo perciben la EA los estudiantes manifiestan:

\section{Tabla 1.}

Percepción respecto a la Educación Ambiental.

\begin{tabular}{lccc}
\hline \multicolumn{1}{c}{ CONCEPTO } & $\mathbf{N}^{\circ}$ & $\%$ \\
\hline $\begin{array}{l}\text { Manera de enseñarnos a cuidar el medio } \\
\text { ambiente }\end{array}$ & 7 & $43.7 \%$ \\
\hline $\begin{array}{l}\text { Conciencia ecológica para proteger el } \\
\text { medioambiente }\end{array}$ & 5 & $31.2 \%$ \\
\hline Sentido de pertenencia por el entorno & 1 & $6.3 \%$ \\
\hline No sabe No responde & 2 & $12.5 \%$ \\
\hline Cambiar actitudes y conductas ambientales & 1 & $6.3 \%$ \\
\hline TOTAL & 16 & $100 \%$ \\
\hline
\end{tabular}

Elaboración: Los autores.

Considerando los datos presentados en la tabla 1 , se tiene que el $43.7 \%$ de los estudiantes perciben la EA como una forma para aprender a cuidar el ambiente, el 31.2\% como espacio para crear conciencia ecológica a fin de protegerlo y $12.6 \%$ como ámbito para desarrollas sentido de pertenencia entorno y generar cambios de actitudes y conductas ambientales, en contraposición al $2 \%$ de los estudiantes que manifiestan no 
saber o no responden. Aunque son diversos los conceptos de los estudiantes es perceptible la coherencia sobre la importancia de aprender a proteger el medio ambiente, cambiando incluso algunas de sus actitudes y comportamientos, que los lleve a tener una conciencia ecológica acorde a las políticas de cuidado con el entorno y la prevención de los posibles riesgos ambientales.

En relación al significado que le dan los estudiantes a la Cultura Ambiental, se tiene:

\section{Tabla 2.}

Significado que le dan a la Cultura Ambiental.

\begin{tabular}{lcc}
\hline \multicolumn{1}{c}{ CONCEPTO } & $\mathbf{N}^{\circ}$ & $\%$ \\
\hline $\begin{array}{l}\text { No arrojar basuras en las calles y ríos y } \\
\text { reciclar }\end{array}$ & 1 & $6.3 \%$ \\
\hline Cuidar el entorno para prevenir enfermedades & 1 & $6.3 \%$ \\
\hline No arrojar basuras & 3 & $18.6 \%$ \\
\hline Es una actividad nueva en la comunidad & 1 & $6.3 \%$ \\
\hline No sabe No responde & 2 & $12.5 \%$ \\
\hline No quemar basuras ni talar arboles & 1 & $6.3 \%$ \\
\hline Mejorar la conciencia ambiental & 7 & $43.7 \%$ \\
\hline TOTAL & 16 & $100 \%$ \\
\hline
\end{tabular}

Elaboración: Los autores.

Al realizar el análisis de los datos de la tabla 2, concerniente al significado que los estudiantes le dan a la Cultura Ambiental, se tiene que el 43.7 la simbolizan como mejora de la conciencia ambiental y el $37.5 \%$ la expresan asociada a no arrojar y quemar basura, no talar árboles y reciclar. Es decir, la significan tanto como conocimiento, como hacer práctico. 
Con respecto al concepto de Movilidad sostenible que tienen los estudiantes se tiene

\section{Tabla 3.}

Concepto de Movilidad Sostenible.

\begin{tabular}{lcc}
\hline \multicolumn{1}{c}{ CONCEPTO } & $\mathbf{N}^{\circ}$ & $\%$ \\
\hline No sabe No responde & 10 & $62.5 \%$ \\
\hline Es transportarse en bicicleta & 5 & $31.25 \%$ \\
\hline $\begin{array}{l}\text { Es una forma de transportarse sin contaminar } \\
\text { el ambiente }\end{array}$ & 1 & $6,25 \%$ \\
\hline TOTAL & 16 & $100 \%$ \\
\hline
\end{tabular}

Elaboración: Los autores.

El análisis de los datos con respecto al concepto "movilidad sostenible" que se presentan en la tabla 3 , evidenciándose que el $62.5 \%$ de los estudiantes manifiestan no sabe a lo que refiere, un $31.25 \%$ asocia la movilidad sostenible con transportarse únicamente en bicicleta, y tan solo el $6.3 \%$ declaran que es un tipo de transporte que no contamina el ambiente.

Otro aspecto importante está relacionado con el conocimiento que tienen los estudiantes sobre los senderos ecológicos, al respecto los estudiantes revelaron: 
Tabla 4.

Conocimiento sobre los senderos ecológicos.

\begin{tabular}{|lcc|}
\hline \multicolumn{1}{c}{ CONCEPTO } & $\mathbf{N}^{\circ}$ & $\%$ \\
\hline Es una ruta de árboles cuidados & 1 & $6.3 \%$ \\
\hline Es un paseo por el bosque & 1 & $6.3 \%$ \\
\hline Zona para deporte y recreación & 5 & $31.2 \%$ \\
\hline Espacio para apreciar la naturaleza & 3 & $18.6 \%$ \\
\hline Espacio para caminar & 5 & $31.2 \%$ \\
\hline No sabe - No responde & 1 & $6.3 \%$ \\
\hline TOTAL & 16 & $99.9 \%$ \\
\hline
\end{tabular}

Elaboración: Los autores.

Los datos que se presentan en la tabla 4, expresan que el $31.2 \%$ de los estudiantes conceptualizan los Senderos Ecológicos como zonas para hacer deporte y recreación, en tanto que el 62.5 la asocian a rutas en los bosque y espacios naturales para paseos y caminatas.

También, fue necesario analizar cuáles son las amenazas más puntuales que perciben los estudiantes respecto a las áreas ambientales importantes de su entorno inmediato, (senderos escolares, rutas ecológicas, ríos y quebradas). En este sentido, lo estudiantes declararon: 


\section{Tabla 5.}

Amenazas puntuales sobre el medio ambiente.

\begin{tabular}{lccc}
\hline \multicolumn{1}{c}{ CONCEPTO } & $\mathbf{N}^{\circ}$ & $\%$ \\
\hline $\begin{array}{l}\text { Cuando se pasa por el caño Bugre el impacto es } \\
\text { negativo (todo tipo de basuras) }\end{array}$ & 8 & $50 \%$ \\
\hline La orilla del rio está muy contaminada & 1 & $6.3 \%$ \\
\hline En mi comunidad no hay ciclo ruta & 1 & $6.3 \%$ \\
\hline Las calles y callejones están descuidados & 1 & $6.3 \%$ \\
\hline Irrespeto de la comunidad con el medio ambiente & 1 & $6.3 \%$ \\
\hline No sabe no responde & 4 & $24.8 \%$ \\
\hline TOTAL & 16 & $100 \%$ \\
\hline
\end{tabular}

Elaboración: Los autores.

Al realizar el análisis de los datos referidos en la tabla 5 en torno a las amenazas más puntuales que perciben los estudiantes respecto a las áreas ambientales emblemáticas de su hábitat inmediato, se tiene que el 56.3\% manifiestan que el impacto cuando cruzan por el Caño Bugre, es altamente negativo debido al grado de contaminación que presenta, por el depósito de todo tipo de basuras que se evidencian en las orillas del rio. En lo que refiere a los beneficios que traería a la comunidad la movilidad sostenible como propuesta pedagógica desde la Educación Física para fortalecer la Educación Ambiental, los estudiantes emitieron diversos conceptos como se aprecia a continuación. 


\section{Tabla 6.}

Beneficios de los ciclo-paseos como herramienta pedagógica transversal de movilidad sostenible para el fortalecimiento de la Cultura Ambiental desde la EF.

\begin{tabular}{lccc}
\hline \multicolumn{1}{c}{ CONCEPTO } & $\mathbf{N}^{\circ}$ & $\%$ \\
\hline $\begin{array}{l}\text { Ayuda a mejorar la condición del aire } \\
\begin{array}{l}\text { Espacio de recreación y deporte para la } \\
\text { familia }\end{array}\end{array}$ & 3 & $18.75 \%$ \\
\hline $\begin{array}{l}\text { Espacio sano para interactuar con la } \\
\text { naturaleza }\end{array}$ & 5 & $31.2 \%$ \\
\hline Permite tranquilidad física y mental & 2 & $12.5 \%$ \\
\hline Se mejora la calidad de vida & 2 & $12.5 \%$ \\
\hline No sabe no responde & 1 & $6.3 \%$ \\
\hline TOTAL & 16 & $99.8 \%$ \\
\hline
\end{tabular}

Elaboración: Los autores.

Los resultados presentados en la tabla 6, refieren que el $93.7 \%$ de los estudiantes consideran beneficioso para la Cultura Ambiental, el desarrollo de los ciclo-paseos desde la EF como herramienta pedagógica, con base en el principio de la movilidad sostenible. Finalmente, se quiso conocer las percepciones de los estudiantes con respecto al uso de la bicicleta, como elemento importante involucrado en el concepto de movilidad sostenible. Al respecto, los estudiantes indican: 


\section{Tabla 7.}

Percepciones del estudiante sobre la bicicleta como medio de movilidad sostenible.

\begin{tabular}{lcc}
\hline \multicolumn{1}{c}{ CONCEPTO } & CANT & $\%$ \\
\hline Para salir a pasear y llegar a la escuela & 2 & $12.5 \%$ \\
\hline Para hacer deporte y recreación & 8 & $50.0 \%$ \\
\hline Para salir a apreciar la naturaleza & 1 & $6.3 \%$ \\
\hline No sabe o no responde & 1 & $6.3 \%$ \\
\hline Para hacer mandados & 4 & $24.8 \%$ \\
\hline Total & 16 & $99.9 \%$ \\
\hline
\end{tabular}

Elaboración: Los autores.

El análisis de los datos que están contenidos en la tabla $N^{\circ} 7$, respecto al uso de la bicicleta como medio de movilidad sostenible, reflejan que el $56.3 \%$ de los estudiantes la perciben como medio útil para hacer deporte y recreación en interacción con la naturaleza, mientras que el $37.3 \%$ manifiestan que es significativa para realizar actividades cotidianas como: salir a pasear, servir de transporte escolar y hacer mandados.

\section{DISCUSIÓN}

Este apartado se presenta como espacio para discutir el significado de los resultados de la investigación y compararlos con los de otros autores. En tal sentido, se tiene que los hallazgos revelan que, existe un alto nivel de desconocimiento sobre dicho tema, tal como lo expresan el $62.5 \%$ de los estudiantes participantes de la investigación.

De allí que pueda decirse que esta situación es un elemento que justifica la necesidad de concientizar a estudiantes y comunidad en general sobre la importancia que tiene la movilidad sostenible mediante el uso de transportes no contaminantes del entorno y del 


\section{CIENCIAMATRIA}

Revista Interdisciplinaria de Humanidades, Educación, Ciencia y Tecnología

Año VII. Vol. VII. N¹. Edición Especial. 2021

Hecho el depósito de ley: pp201602FA4721

ISSN-L: 2542-3029; ISSN: 2610-802X

Universidad Nacional Experimental Francisco de Miranda (UNEFM). Santa Ana de Coro. Venezuela

Ángel Darío Díaz-Cordero; José Francisco Marimón-Lozano; María del Carmen Jiménez-Barriosnuevo

medio ambiente, lo que permite mejoras en la calidad de vida de las personas, al igual que lo expresan Herrera (2018) \& Sanz (2016).

De manera sintetizada, los datos reportan el $87.5 \%$ de los estudiantes consideran la EA como un espacio educativo para desarrollar conciencia ecológica y sentido de pertenecía por el entorno, a propósito de aprender nuevas actitudes y conductas de cuidado del medio ambiente. Planteamiento que coincide con lo expresado por Pulido \& Olivera (2018), Wilches, (2013) y Torres \& Velandia (2013), a razón de que la EA es una estrategia que permite las relaciones de interdependencia ser humano-naturaleza, a través de la experiencia de problematización de la realidad, que conlleven al desarrollo de capacidades y transformación de comportamientos que atiendan al cuidado del medio ambiente y el entorno. Hallazgo que se afianza, si se correlaciona con que el $81.2 \%$ de los estudiantes significan la Cultura Ambiental como conciencia y cuidado del entorno mediante conductas concretas para prevenir la contaminación de los espacios ambientales y el entorno en general.

Cabe resaltar, la preeminente necesidad que existe de promover una Cultura Ambiental en pro del cuidado del entorno, ya que los datos ponen en evidencia el impacto que generan los sitios naturales emblemáticos y espacios de la ciudad dado los altos niveles de contaminación que presentan. Tal es el caso de Caño Bugre, rio con trascendencia ambiental, pues, cuenta con una extensión aproximada de 33 kilómetros trayecto que recorre los municipios de Montería, Cereté, San Pelayo, Cotorra, Chimá y Lorica, hasta descargar su caudal de agua en la Ciénaga Grande de Lorica.

Promover esta Cultura Ambiental basada en la movilidad sostenible, se puede concretar mediante la formación que se imparte a través de la EF. Es decir, desarrollar el conocimiento ambiental, de manera transversalizada con las actividades físico-deportivas y recreativas, mediante el uso de la bicicleta como medio de movilidad sostenible de uso cotidiano y para el desarrollo de los ciclopaseos en senderos ecológicos. Pues esto, aparte de concretarse como un espacio para el deporte y la recreación en interacción con 
la naturaleza, coadyuva a mejorar la condición del aire y las condiciones físicas y mentales y con ello la calidad de vida de los ciudadanos; ya que es necesario reconocer que la salud y el bienestar de toda una comunidad están estrechamente relacionados con la calidad y cuido del medio ambiente.

Hallazgo que se compagina con lo enunciado por Luque (2016) \& Navarro (2014), en relación a el uso de la bicicleta en el desarrollo de ciclo-paseos posibilita la formación ciudadana para reconocer los entornos naturales, favorece el desarrollo de actitudes y hábitos saludables en relación con la salud física y mental, ya que se potencia el desarrollo de valores para la convivencia positiva entre las personas, los ecosistemas y su entorno inmediato.

Para cerrar, todos estos resultados ponen en evidencia, tal como expresa Flórez (2012), la necesidad de desplegar la EA como eje transversalizador de la formación, que se ha de concretar a través de programas y proyectos ambientales articulados a toda la estructura curricular y al PEI.

\section{CONCLUSIONES}

Es de recordar que con el estudio se buscó saber cómo perciben la movilidad sostenible, los estudiantes de la Institución Educativa "El Carmen" en Cotorra, Departamento de Córdoba - Colombia. Esto a razón de que existe la necesidad de desarrollar una Cultura Ambiental desde una nueva visión de los programas de Educación Ambiental que se conciban y ejecuten de manera transversalizada e integrada a otros programas curriculares como el de Educación Física. Tomando aquí un papel preponderante la movilidad sostenible, donde el uso de la bicicleta y los ciclopaseos se constituyen en las principales herramientas para ello. para el aprendizaje y el desarrollo de conductas, comportamientos y acciones de cuidado del medio ambiente y de los entornos donde se hace vida, a la par del desarrollo de actividades físico-deportivas y recreativas. 
Atendiendo a esto, con base en los resultados se puede mostrar los siguientes señalamientos:

- Existe desconocimiento en cuanto al concepto de movilidad sostenible y todo lo que ello involucra, aunque se tenga información que es un tema asociado a la práctica y uso de la bicicleta.

- La Educación Ambiental representa en los contextos educativos y curriculares un ámbito para la formación transversalizada y el desarrollo de la cultura del cuidado del entorno y de los espacios naturales emblemáticos de los territorios geográficos, impactados por los efectos adversos de los distintos tipos de contaminación que prevalecen en el medio ambiente.

- La Educación Física se constituye en un programa académico curricular propicio para desplegar procesos formativos transversalizados e integrados a la Educación Ambiental asumiendo el concepto de movilidad sostenible.

Hechas las consideraciones anteriores, se deduce que la movilidad sostenible se constituye en un enfoque pedagógico innovador y provechoso que puede ser implementado por el PRAE de las instituciones educativas, para el desarrollo de la Cultura Ambiental. Asimismo, puede concretarse transversalmente desde la Educación Física, mediante el desarrollo de ciclopaseos y el uso de la bicicleta; pues, estas herramientas asociadas a dicho concepto, son idóneas para la formación de ciudadanos con conciencia ambiental, el aprendizaje y el desarrollo de conductas, comportamientos y acciones de cuidado ecológico y del medio ambiente, entrelazada al ejercicio físico-deportivo y la actividad recreativa que se realizan en espacios naturales y entornos donde se hace vida cotidiana. 


\section{REFERENCIAS}

Arellana, J., Puche, K. \& García S. (2019). Propuesta de política pública de movilidad. Recuperado de: https://n9.cl/69s3i

Baena \& Granero, A. (2008). Las actividades físicas en la naturaleza en el currículum actual: contribución a la educación para la ciudadanía y los derechos humanos. Sistema de Información Científica. Red de Revistas Científicas de América Latina, el Caribe, España y Portugal Nuevas tendencias en Educación, Recuperado de https://www.redalyc.org/pdf/3457/345732279008.pdf

De Maio, M., Linório, M. \& Tûrk, S. (2018). Esporte e meio ambiente: a disciplina de esporte e gestão ambiental (EGA), na formação de alunos de Educação Física, na região do sertão nordestino. Revista Motrivivência. 30(56). Recuperado de: https://n9.cl/ntl86

Flórez, A. (2012). La educación ambiental: un apuesta hacia la integración escuela comunidad. Revista Praxis y Saber. 3(5). 79-101. DOI:10.19053/22160159.1135

González, M. (2014). Evaluación de los ambientes mixtos de aprendizaje desde la perspectiva del estudiante. Revista Iberoamericana para la Investigación y el Desarrollo Educativo, 12 (1). Recuperado de https://n9.cl/ncnb6

Herrera, J. (2018). Análisis sobre la implementación de movilidad sostenible en zonas urbanas. (Trabajo de grado). Universidad Cooperativa de Colombia, Santa Marta, Colombia. Recuperado de https://n9.cl/t07e4

Lagardera, F. (2009). Educación física y sostenibilidad. Asociación Científico Cultural en Actividad Física y Deporte (ACCAFIDE). 3(1), 26-28. Recuperado de: https://n9.cl//3k1j

Legido, J., Ruiz, M., Brito, R. \& Navarro, A (2008). Deporte y medio ambiente. Origen, significado, composición y carácter redundante de la expresión medio ambiente. Hospital Universitario Insular de Gran Canaria. Universidad de Las Palmas de Gran Canaria. 5(15), 9. Recuperado de https://n9.cl//3k1j

Luque, P. (2016). La movilidad urbana sostenible una nueva razón para fomentar el uso de la bicicleta en el ámbito educativo. Revista digital de Educación Física. 40 (1), 36-50. https://dialnet.unirioja.es/descarga/articulo/5456612.pdf 
Luque, P., Baena, A. \& Granero, A. (2011). Buenas prácticas para un desarrollo sostenible en los eventos deportivos en el medio natural. Interciencia. Sistema de Información Científica. Red de Revistas Científicas de América Latina, el Caribe, España y Portugal 36(7), 531-537. Recuperado de: https://www.redalyc.org/pdf/339/33919424009.pdf

Martínez, J. (2017). Fundamentos de la Educación Ambiental. Recuperado de https://www.unescoetxea.org/ext/manual/html/fundamentos.html

Ministerio de Educación Nacional (MEN) \& Ministerio de Ambiente y Desarrollo Sostenible (MADS) (2002). Política Nacional de Educación Ambiental. Bogotá: Cooperativa Editorial. Recuperado de https://n9.cl/ca22

Minambiente. (2002). Programa para el manejo sostenible y restauración de ecosistemas de Medio ambiente de montaña en Colombia. Recuperado de https://n9.cl/rth6

Navarro L. (2014). La bicicleta como recurso interdisciplinar. Universidad de Valladolid. Recuperado de: http://uvadoc.uva.es/handle/10324/590

Nyambe, C. (2012). Relación Educación Física-deporte y medio ambiente; ¿tierra incógnita? Revista Monteverdia. 5(2), 1-7. Recuperado de https://revistas.reduc.edu.cu/index.php/monteverdia/article/view/1881

Palella, S. \& Martins, F. (2015). Metodología de la Investigación Cuantitativa. Caracas. FEDUPEL.

Páramo, P. (2009). Pedagogía urbana: elementos para su delimitación como campo de conocimiento. Revista Colombiana de Educación. 57 (1). Recuperado de https://doi.org/10.17227/01203916.7586

Pulido, V. \& Olivera, E. (2018). Aportes pedagógicos a la Educación Ambiental: una perspectiva teórica. Revista de Investigaciones Altoandinas, 20 (3) 333-346. Recuperado de http://dx.doi.org/10.18271/ria.2018.397

Sáez, J. (2012). Las actividades físico-deportivas en el medio natural como contenido dentro del sistema educativo español. Desarrollo y evolución de las leyes educativas. Revista Pedagógica ADAL, 25 (1), 33-37. https://dialnet.unirioja.es/servlet/articulo?codigo $=5337900$ 
Revista Interdisciplinaria de Humanidades, Educación, Ciencia y Tecnología

Año VII. Vol. VII. N¹. Edición Especial. 2021

Hecho el depósito de ley: pp201602FA4721

ISSN-L: 2542-3029; ISSN: 2610-802X

Universidad Nacional Experimental Francisco de Miranda (UNEFM). Santa Ana de Coro. Venezuela

Ángel Darío Díaz-Cordero; José Francisco Marimón-Lozano; María del Carmen Jiménez-Barriosnuevo

Sanz, A. (2016). Cómo lograr una unidad didáctica sobre el uso de la bicicleta en una escuela urbana de Segovia. Universidad de Valladolid. Recuperado de: http://uvadoc.uva.es/handle/10324/17513

Torres, J. \& Velandia, S. (2013). Influencia de las estrategias pedagógicas en los procesos de aprendizaje de los estudiantes de una institución de básica primaria de la ciudad de Bucaramanga. Universidad Pontificia Bolivariana. Recuperado de https://n9.cl/8undk

Wilches, G. (2013). Brújula, bastón y lámpara para trasegar los caminos de la Educación Ambiental. Bogotá, Colombia. Recuperado de. https://n9.cl/hiz7u

(C2021 por los autores. Este artículo es de acceso abierto y distribuido según los términos y condiciones de la licencia Creative Commons Atribución-NoComercial-Compartirlgual 4.0 Internacional (CC BY-NC-SA 4.0)

(https://creativecommons.org/licenses/by-nc-sa/4.0/). 